\title{
Comparison of single-injection ultrasound-guided approach versus multilevel landmark-based approach for thoracic paravertebral blockade for breast tumor resection: a retrospective analysis at a tertiary care teaching institution
}

This article was published in the following Dove Press journal: Journal of Pain Research

28 June 2017

Number of times this article has been viewed

\author{
Jagroop Singh Saran' \\ Amie L Hoefnagel' \\ Kristin A Skinner ${ }^{2}$ \\ Changyong Feng ${ }^{3}$ \\ Daryl Irving Smith'
}

'Acute Pain Service, Department of Anesthesiology, University of Rochester School of Medicine and Dentistry, ${ }^{2}$ Department of Surgical Oncology, University of Rochester School of Medicine and Dentistry, University of Rochester Medical Center, ${ }^{3}$ Department of Biostatistics and Computational Biology, University of Rochester School of Medicine and Dentistry, Rochester, NY, USA
Correspondence: Daryl Irving Smith Acute Pain Service, Department of Anesthesiology, University of Rochester School of Medicine and Dentistry, 60 I Elmwood Avenue, Box 604, Rochester, NY 14642, USA

Tel +I 5852763770

Fax + I 585244727 |

Email Daryl_Smith@URMC.rochester.edu
Background: The role of thoracic paravertebral blockade (TPVB) in decreasing opioid requirements in breast cancer surgery is well documented, and there is mounting evidence that this may improve survival and reduce the rate of malignancy recurrence following cancer-related mastectomy. We compared the two techniques currently in use at our institution, the anatomic landmark-guided (ALG) multilevel versus an ultrasound-guided (USG) single injection, to determine an optimal technique.

Methods: We retrospectively reviewed records of patients who received TPVB from January 2013 to December 2014. Perioperative opioid use, post anesthesia care unit (PACU) pain scores and length of stay, block performance, and complications were compared between the two groups. Results: We found no statistical difference between the two approaches in the studied outcomes. We did find that the number of times attending physicians in the ALG group took over the blocks from residents was significantly greater than that of the USG group $(p=0.006)$ and more local anesthetic was used in the USG group ( $p=0.04$ ).

Conclusion: This study compared the ALG approach with the USG approach for patients undergoing mastectomy for breast cancer. Based on our observations, an attending physician is more likely to take over an ALG injection, and more local anesthetic is administered during USG single injection.

Keywords: thoracic paravertebral block, regional anesthesia, mastectomy, breast cancer

\section{Introduction}

The American Cancer Society predicts that there will be 231,840 new cases of invasive breast cancer diagnosed in the US in $2015 .{ }^{1}$ Given that many of these patients will undergo mastectomy, considerable research has been done on anesthetic techniques. The benefits of regional anesthesia techniques, including thoracic paravertebral blockade (TPVB), include improved analgesia, reduced opioid consumption, reduced postoperative nausea and vomiting. ${ }^{2-6}$ TPVB offers several advantages for breast cancer surgery as an opioid sparing technique, including mounting evidence supporting reduced tumor recurrence following mastectomy. ${ }^{7-9}$ There is also evidence that a paravertebral block, either a single shot or continuous catheter, can lower the incidence of chronic pain following mastectomy. ${ }^{10-13}$ While there are variations on the techniques for placement of a paravertebral block, the two commonly used techniques are ultrasound guided 
(USG) and landmark based. ${ }^{14}$ It is our standard practice to offer patients undergoing mastectomy paravertebral nerve blockade preoperatively. Here, we compared the two primary techniques used at our institution, USG and anatomic landmark guided (ALG), to see if one technique offers a higher success rate. We defined success based on the patient's postoperative pain and overall opioid use. We also looked at time for block performance and length of stay in post anesthesia care unit (PACU) as additional primary end points.

\section{Methods}

The institutional review board (IRB) at the University of Rochester School of Medicine approved this single-center retrospective study (RSRB: 54877). A waiver of consent was granted by the IRB due to the retrospective nature of data collection as all specified criteria for a waiver of Health Insurance Portability and Accountability Act (HIPAA) of 1996 requirements were met. All study procedures were completed at the University of Rochester Medical Center/ Strong Memorial Hospital, a tertiary care, teaching hospital located in Rochester, NY, between January 2013 and December 2014. The surgical procedures performed included mastectomy, mastectomy with sentinel lymph node biopsy, modified mastectomy, mastectomy with implant insertion, partial mastectomy, partial mastectomy with sentinel lymph node biopsy, and breast biopsy with axillary lymph node dissection. The exclusion criteria included age $<18$ years, noncancer-related mastectomy, body mass index (BMI) $<18 \mathrm{~kg} / \mathrm{m}^{2}$ or $>35 \mathrm{~kg} / \mathrm{m}^{2}$, allergy to opioid medication, and daily opioid use of $>50 \mathrm{mg}$ morphine equivalents. We also excluded incomplete medical records or those with missing data points from our analysis. These exclusion criteria were set at the time of IRB submission and retrospectively applied following completion of all chart reviews. A total of 108 charts were included in the final data set, 53 from the USG group and 55 from the ALG group.

\section{Preoperative procedure}

All patients received premedication for anxiolysis and analgesia with either intravenous (IV) midazolam 1-6 mg and/or IV fentanyl 50-200 $\mu \mathrm{g}$ prior to receiving the block. All patients were monitored with noninvasive blood pressure and pulse oximetry and given supplemental oxygen via nasal cannula at $4 \mathrm{~L} / \mathrm{min}$ during the procedure.

\section{Thoracic paravertebral block technique}

The technique for the PVB was chosen at the discretion of the attending anesthesiologist. In the anatomic or landmark-based approach, the spinous process of $\mathrm{C} 7$ and the lower tips of the scapula (parallel to T7) were identified and used to identify the target levels. The needle insertion site was $2.0-2.5 \mathrm{~cm}$ lateral to the spinous process, where the needle was inserted until contact with the transverse process was made, and then the needle was redirected cephalad until a loss of resistance was felt, generally $<1 \mathrm{~cm}$ past the transverse process. This loss of resistance was the needle passing the superior costotransverse ligament and entering the paravertebral space, which only occurs in the thoracic region. Local anesthetic was then injected into the space, and this process was repeated from $\mathrm{T} 2$ to $\mathrm{T} 7$ as needed to achieve the desired spread based on the surgical procedure.

In the USG approach, an 18-gauge, 10-cm Tuohy needle was introduced using an in-plane approach relative to a highfrequency ultrasound transducer. The needle was advanced in a cephalad manner under direct visualization until the superior costotransverse ligament was punctured and the volume of local anesthetic was deposited at the thoracic interspace $\mathrm{T} 4$.

\section{Intraoperative and postoperative management}

The patients all underwent general anesthesia with the airway and intraoperative management, including opioid and nonopioid analgesia, at the discretion of the anesthesia team. At the conclusion of the surgery, all patients were transferred to the PACU. In the PACU all patients were treated with IV hydromorphone in 0.2-0.4 mg increments for a Visual Analog Scare (VAS) score of 4/10 or greater or patient request for analgesia. Patients were monitored in the PACU until they met phase I discharge criteria.

\section{Outcomes}

Our primary end points were intraoperative opioid use, duration of PACU stay, PACU opioid use, and initial and final PACU phase I pain scores. The cumulative doses of intraoperative and postoperative opioids were converted to IV morphine equivalents.

We collected the time to perform the block (in minutes) and the amount of anxiolytic required (IV midazolam) and analgesic (IV fentanyl) given during block placement. In addition, the amount of local anesthetic used and the number of attempts needed for successful blockade either by resident or attending attempts were also recorded. We also noted any block-related complications, including pneumothorax, inadvertent vascular puncture, intravascular injection, epidural spread, and systemic toxicity. 


\section{Data management and statistical analysis}

Study data were collected and managed using Research Electronic Data Capture (REDCap) electronic data capture tools hosted at University of Rochester School of Medicine and Dentistry. ${ }^{15}$ The Shapiro-Wilk test was used to examine the normality assumption of continuous measurements in the two groups. ${ }^{16}$ Our result failed to reject this hypothesis for all continuous measurements. The two-sample $t$-test was used to compare the mean values of continuous measurements in the two groups. The significance level was set at 0.05. All statistical analyses were implemented with SAS 9.4 (SAS Institute Inc., Cary, NC, USA).

\section{Results}

\section{Study population}

We reviewed a total of 148 records of patients that received a TPVB for breast tumor resection from January 2013 to December 2014 at our institution. Of these 148 records, 40 were excluded because they did not meet the inclusion criteria or because they had incomplete medical records. Of the remaining 108 records, 55 patients received TPVB using the ALG approach and 53 patients using the USG approach. Patient demographic characteristics were similar with no statistically significant differences $(p>0.05)$ between the two groups (Table 1).

\section{Outcome measures}

We did find a statistically significant difference in the amount of local anesthetic used in the USG group (32.32 $\pm 5.67 \mathrm{~mL})$ compared with that used in the ALG group $(30.09 \pm 5.23 \mathrm{~mL}$,

Table I Patient characteristics

\begin{tabular}{|c|c|c|c|c|c|c|c|}
\hline & \multicolumn{3}{|c|}{ ALG } & \multicolumn{3}{|c|}{ USG } & \multirow[t]{2}{*}{$p$-value } \\
\hline & $n$ & Mean & $\begin{array}{l}\text { Std } \\
\text { Dev }\end{array}$ & $\overline{\mathbf{N}}$ & Mean & $\begin{array}{l}\text { Std } \\
\text { Dev }\end{array}$ & \\
\hline Age (years) & 55 & 62.07 & 12.89 & 53 & 61.68 & 11.39 & 0.87 \\
\hline Height (cm) & 55 & 163.83 & 8.3 & 53 & 165.12 & 7.01 & 0.38 \\
\hline Weight (kg) & 55 & 73.08 & 11.47 & 53 & 76.33 & 13.1 & 0.17 \\
\hline $\mathrm{BMI}\left(\mathrm{kg} / \mathrm{m}^{2}\right)$ & 55 & 27.24 & 3.92 & 53 & 28 & 4.44 & 0.35 \\
\hline
\end{tabular}

Abbreviations: ALG, anatomic landmark guided; BMI, body mass index; Std Dev, standard deviation; USG, ultrasound guided. $p=0.04)$. There was no statistically significant difference between the time to complete the block despite the USG technique only requiring a single injection and the ALG approach requiring five injections at the T2-T6 levels (Table 2).

We found no statistically significant differences in our primary outcomes (Table 3). The intraoperative opioid requirement in IV morphine equivalents was $12.21 \pm 8.06 \mathrm{mg}$ in the ALG group and $11.97 \pm 10.38 \mathrm{mg}$ in the USG group $(p=0.89)$. The PACU opioid consumption in IV morphine equivalents was $4.13 \pm 4.22 \mathrm{mg}$ in the ALG group and $4 \pm 4.02 \mathrm{mg}$ in the USG group $(p=0.87)$. The PACU length of stay was $109 \pm 92$ minutes in the ALG group and $120 \pm 177$ minutes in the USG group $(p=0.68)$. The PACU first pain score was $2.76 \pm 3.39$ in the ALG group and 2.15 \pm 2.91 in the USG group $(p=0.32)$. The PACU last pain score was $2.16 \pm 2.18$ in the ALG group and $2.53 \pm 2.2$ in the USG group $(p=0.39)$. The duration of surgery was $133 \pm 44$ minutes in the ALG group and $138 \pm 82$ minutes in the USG group $(p=0.66)$.

There was a statistically significant difference in the number of times that the attending anesthesiologist completed the procedure in the ALG group when compared to the USG group $(p=0.006)$.

\section{Complications}

There was one patient who suffered a pneumothorax in the USG group that was discovered in the PACU and treated conservatively with medical management. There were no complications in the ALG group.

\section{Discussion}

This is the first study to compare TPVB techniques placed for post-operative pain control in patients undergoing mastectomy for cancer. There is mounting evidence in the literature that the choice of anesthetic technique affects incidence rates of recurrence and metastasis after tumor resection..$^{7-9}$ If two or more interventional techniques exist, it is important to determine the optimal opiate-sparing perioperative analgesic regimen, and the superiority of either technique must be sought based upon specific outcomes. Determination and implementation of optimal therapy may ultimately prove

Table 2 Comparison between the groups for TPVB placement

\begin{tabular}{|c|c|c|c|c|c|c|c|}
\hline & \multicolumn{3}{|c|}{ ALG } & \multicolumn{3}{|c|}{ USG } & \multirow[t]{2}{*}{ p-value } \\
\hline & $n$ & Mean & Std Dev & $n$ & Mean & Std Dev & \\
\hline Time to perform the block (minutes) & 55 & 12.04 & 5.99 & 53 & 10.81 & 5.08 & 0.25 \\
\hline $0.5 \%$ bupivacaine with $\mathrm{I}: 200,000$ epinephrine $(\mathrm{mL})$ & 55 & 30.09 & 5.23 & 53 & 32.32 & 5.67 & 0.04 \\
\hline Midazolam (mg) & 55 & 1.76 & 0.58 & 53 & 1.6 & 1.12 & 0.35 \\
\hline Fentanyl (mg) & 55 & 90 & 31.03 & 53 & 92.45 & 30.06 & 0.68 \\
\hline
\end{tabular}

Abbreviations: ALG, anatomic landmark guided; Std Dev, standard deviation; TPVB, thoracic paravertebral blockade; USG, ultrasound guided. 
Table 3 Comparison between the two groups of intraoperative and PACU outcomes

\begin{tabular}{|c|c|c|c|c|c|c|c|}
\hline & \multicolumn{3}{|c|}{ ALG } & \multicolumn{3}{|c|}{ USG } & \multirow[t]{2}{*}{$p$-value } \\
\hline & $\mathbf{n}$ & Mean & Std Dev & $\mathbf{n}$ & Mean & Std Dev & \\
\hline Intraoperative opioid use (IV morphine milligram equivalent) & 55 & 12.21 & 8.06 & 53 & 11.97 & 10.38 & 0.89 \\
\hline Duration of surgery (minutes) & 55 & 133.09 & 44.42 & 53 & 138.77 & 81.78 & 0.66 \\
\hline PACU stay (minutes) & 55 & 109 & 92.85 & 53 & 120.43 & $|76.9|$ & 0.68 \\
\hline PACU first pain score & 55 & 2.76 & 3.39 & 53 & 2.15 & 2.91 & 0.32 \\
\hline PACU last pain score & 55 & 2.16 & 2.18 & 53 & 2.53 & 2.2 & 0.39 \\
\hline PACU opioid use (IV morphine milligram equivalent) & 55 & 4.13 & 4.22 & 53 & 4 & 4.02 & 0.87 \\
\hline
\end{tabular}

Abbreviations: ALG, anatomic landmark guided; IV, intravenous; PACU, post anesthesia care unit; Std Dev, standard deviation; TPVB, thoracic paravertebral blockade; USG, ultrasound guided.

to have beneficial effects upon patient survival. There were no significant differences between the two techniques when intraoperative opioid use, duration of PACU stay, PACU opioid use, and pain score in PACU as markers for block success were compared. Similarly, we found no significant differences in the time to complete the blocks, despite the single-injection technique used with the USG method versus the multiple injections used with the ALG techniques. The data did reveal that trainees were allowed to complete the block in its entirety more often when USG is used.

One complication did occur during the 2-year period examined: one patient developed pneumothorax in the USG group. There was no incidence of local anesthetic toxicity, epidural or subarachnoid injection, hypotension, or bradycardia in either group. A recent large retrospective review of $>1400$ TPVB injections revealed a complication rate of $0.7 \%$, including symptomatic bradycardia and hypotension, vasovagal episodes, and local anesthetic toxicity. ${ }^{17}$

The observed significant difference in the number of procedures completed by the attending anesthesiologists between the two groups deserves examination. This difference may exist because of the immediate visual feedback available to attending anesthesiologists when using the ultrasound technique. Visual feedback in real time may provide more security to the instructor, which in turn results in more leeway for the trainee. A brief review of the techniques used in the performance of the individual block modalities may provide further clarification. In the landmark technique, the needle tip is advanced without visual confirmation of its location. The transverse process is contacted, and then the needle is withdrawn, redirected, "walked off" the transverse process, and redirected toward the paravertebral space. This may yield a perceived loss of resistance or a "pop" that is transmitted along the needle and "felt" by the operator. However, this "pop" is not reliable, and its discernment may be even less reliably sensed by the inexperienced trainee. Medial and lateral directional variations are also difficult to discern.
These may decrease the rate of success of the block if the needle tip courses lateral to the intercostal space and the injectate is deposited in that location, or the needle may be directed medially and acquire the epidural space with resultant, inadvertent injection into the epidural space. Injection at this site might not only yield a more profound blockade but also impose a greater risk of undesired motor and cardiovascular effects. Finally and perhaps of greatest concern to both the instructor and trainee is the variable distance between the superior costotransverse ligament and the parietal pleura. The approach to this space can be unsettling for some operators since it requires greater three-dimensional thinking and precise manual adjustments and readjustments to accomplish the block. This may prove daunting to some inexperienced trainees. It should be noted here that these blocks are frequently performed in the immediate preoperative setting. Here there is often little time for trainees to err, make corrections, and then reattempt blocks when there is pressure to stay on schedule and move patients toward their surgical incisions. The number of trainee attempts may be severely limited before instructor intervention is deemed appropriate. Conversely, the USG technique may provide teaching benefits not found with the anatomic landmark method. Perhaps the foremost teaching benefit is the ability to visualize the needle tip continuously. This may provide the greatest consolation to trainee and operator alike. Determination of laterality of the target zone is possible because of the distinct ultrasonographic anatomy of the ribs versus that of the transverse processes. Needle depth determination is not based upon "usual" distances but actual observation of the approach to critical structures and targets. Successful needle tip positioning in the paravertebral space can be confirmed by direct visualization deep to the superior costotransverse ligament and then re-confirmed by anterior displacement of the pleura upon injection. Appropriate positioning may also be confirmed by observing injectate spread to the paravertebral spaces in both the cephalad and caudad directions. While 
no studies regarding specific comfort level of instructors or trainees performing these blocks have been conducted, there appears to be more objective and directly observable confirmation of proper procedure maneuvers available in the USG technique. Whether this renders one technique overall superior to another remains to be seen, but with respect to the hands-on repetitions needed to gain proficiency in a procedure at the trainee level. ${ }^{18-20}$

There are several limitations in our study. This was a retrospective review of medical charts and thus there was a lack of randomization. The technique of TPVB, either anatomic or USG, was not randomized and was at the discretion of the anesthesiologist. The opioid regimen was not standardized and was also at the discretion of the anesthesiologist. We included many different types of surgeries in our analysis, and not all surgeries were equally as painful. There were some surgical procedures that had longer operative times and other procedures that required more analgesia. However, the types of surgeries were equally represented in both groups, thus allowing us to average our end points and compare them. Additionally, all the surgeries in this study were performed by one surgeon, further controlling for surgical pain and operative time. We did not account for differences in the training levels of the residents performing the block and assumed that the postgraduate level was uniform in the two groups. We found that the attending anesthesiologist was more likely to complete the procedure in the landmark-based approach and assumed that the efficacy was similar between resident anesthesiologists and attending anesthesiologists. Terkawi et $\mathrm{al}^{21}$ found in their meta-analysis that a multilevel block improves pain control with movement in the first several hours following surgery but is associated with increased risk of procedural complications such as inadvertent vascular puncture. We did not see these differences in our retrospective review. However, the amount of local anesthetic used was significantly higher in the ultrasound group that used a single injection technique, which likely resulted in similar spread of local anesthetic and therefore similar analgesia between the two techniques.

\section{Conclusion}

Retrospective review of patients who received TPB either using multiple-injection anatomic landmark-based approach or single-injection USG approach at our institution undergoing tumor resection for breast cancer revealed no difference in perioperative opioid use and PACU pain scores. Prospective randomized trials are needed to determine if either technique provides superior efficacy with decreased complication rates.
Furthermore, the role of trainees in performing this block needs to be explored.

\section{Acknowledgment}

This study was presented as a scientific poster at the International Anesthesia Research Society Annual Meeting, May 2016, San Francisco, CA, USA.

\section{Disclosure}

The authors report no conflicts of interest in this work.

\section{References}

1. American Cancer Society. Breast Cancer Facts \& Figures 2015-2016. Atlanta, GA: American Cancer Society, Inc; 2015.

2. Karmakar MK. Thoracic paravertebral block. Anesthesiology. 2001;95(3):771-780.

3. Karmakar MK, Samy W, Li JW, et al. Thoracic paravertebral block and its effects on chronic pain and health-related quality of life after modified radical mastectomy. Reg Anesth Pain Med. 2014;39(4):289-298.

4. Klein SM, Bergh A, Steele SM, Georgiade GS, Greengrass RA. Thoracic paravertebral block for breast surgery. Anesth Analg. 2000;90(6):1402-1405.

5. Hara K, Sakura S, Nomura T, Saito Y. Ultrasound guided thoracic paravertebral block in breast surgery. Anaesthesia. 2009;64(2):223-225.

6. Fahy AS, Jakub JW, Dy BM, et al. Paravertebral blocks in patients undergoing mastectomy with or without immediate reconstruction provides improved pain control and decreased postoperative nausea and vomiting. Ann Surg Oncol. 2014;21(10):3284-3289.

7. Yeager MP, Rosenkranz KM. Cancer recurrence after surgery: a role for regional anesthesia? Reg Anesth Pain Med. 2010;35(6):483-484.

8. Forget $\mathrm{P}$, Vandenhende J, Berliere $\mathrm{M}$, et al. Do intraoperative analgesics influence breast cancer recurrence after mastectomy? A retrospective analysis. Anesth Analg. 2010;110(6):1630-1635.

9. Bortsov AV, Millikan RC, Belfer I, Boortz-Marx RL, Arora H, McLean SA. $\mu$-Opioid receptor gene A118G polymorphism predicts survival in patients with breast cancer. Anesthesiology. 2012;116(4):896-902.

10. Andreae MH, Andreae DA. Regional anaesthesia to prevent chronic pain after surgery: a Cochrane systematic review and meta-analysis. Br J Anaesth. 2013;111(5):711-720.

11. Kairaluoma PM, Bachmann MS, Rosenberg PH, Pere PJ. Preincisional paravertebral block reduces the prevalence of chronic pain after breast surgery. Anesth Analg. 2006;103(3):703-708.

12. Ilfeld BM, Madison SJ, Suresh PJ, et al. Treatment of postmastectomy pain with ambulatory continuous paravertebral nerve blocks: a randomized, triple-masked, placebo-controlled study. Reg Anesth Pain Med. 2014;39(2):89-96.

13. Ilfeld BM, Madison SJ, Suresh PJ, et al. Persistent postmastectomy pain and pain-related physical and emotional functioning with and without a continuous paravertebral nerve block: a prospective 1-year follow-up assessment of a randomized, triple-masked, placebo-controlled study. Ann Surg Oncol. 2015;22(6):2017-2025.

14. Batra RK, Krishnan K, Agarwal A. Paravertebral block. J Anaesthesiol Clin Pharmacol. 2011;27(1):5-11.

15. Harris PA, Taylor R, Thielke R, Payne J, Gonzalez N, Conde JG. Research electronic data capture (REDCap) - a metadata-driven methodology and workflow process for providing translational research informatics support. J Biomed Inform. 2009;42(2):377-381.

16. Shapiro SS, Wilk MB. An analysis of variance test for normality (complete samples). Biometrika. 1965;52(3/4):591-611.

17. Pace MM, Sharma B, Anderson-Dam J, Fleischmann K, Warren L, Stefanovich P. Ultrasound-guided thoracic paravertebral blockade: a retrospective study of the incidence of complications. Anesth Analg. 2016; 122(4):1186-1191. 
18. Konrad C, Schupfer G, Wietlisbach M, Gerber H. Learning manual skills in anesthesiology: is there a recommended number of cases for anesthetic procedures? Anesth Analg. 1998;86(3):635-639.

19. Orebaugh SL, Bigeleisen PE, Kentor ML. Impact of a regional anesthesia rotation on ultrasonographic identification of anatomic structures by anesthesiology residents. Acta Anaesthesiol Scand. 2009;53(3):364-368.
20. Kopacz DJ, Neal JM, Pollock JE. The regional anesthesia "learning curve". What is the minimum number of epidural and spinal blocks to reach consistency? Reg Anesth. 1996;21(3):182-190.

21. Terkawi AS, Tsang S, Sessler DI, et al. Improving analgesic efficacy and safety of thoracic paravertebral block for breast surgery: a mixedeffects meta-analysis. Pain Physician. 2015;18(5):E757-E780.

\section{Publish your work in this journal}

The Journal of Pain Research is an international, peer reviewed, open access, online journal that welcomes laboratory and clinical findings in the fields of pain research and the prevention and management of pain. Original research, reviews, symposium reports, hypothesis formation and commentaries are all considered for publication.
Dovepress

The manuscript management system is completely online and includes a very quick and fair peer-review system, which is all easy to use. Visit http://www.dovepress.com/testimonials.php to read real quotes from published authors. 